\title{
Diagnosis of micrometastasis in muscle invasive bladder cancer through immunohistochemistry analysis: is there indication for routine evaluation?
}

\author{
Gustavo Cuck, Isabela Werneck da Cunha* (D), Walter Henriques da Costa*, Clóvis Antônio Lopes Pinto, \\ Carlos Alberto Ricetto Sacomani, Francisco Paulo da Fonseca and Gustavo Cardoso Guimarães
}

\begin{abstract}
Background: Muscle invasive bladder cancer (BC) has a mortality rate of 50\% in 5 years, despite the aggressive treatments currently used. The diagnosis of latent tumor cells in histologically normal lymph nodes (LN) may have prognostic value and may explain the tumoral recurrence in BC.

Methods: Here we evaluated the use of the AE1 AE3 cytokeratin marker through immunohistochemical examination of LNs to diagnose micrometastasis in patients with BC undergoing radical cystectomy (RC) and lymph node dissection. Sixty-one patients with pNO diseases who were submitted to RC were studied. Conventional histological evaluation indicated that these patients did not have lymph node metastasis. Histological sections were reviewed and analyzed by immunohistochemistry $(\mathrm{IHC})$ using the AE1AE3 antibody in single sections.

Results: The total number of removed LNs was 832, averaging $13.64 \mathrm{LNs}$ per patient. The IHC evaluation revealed that LN from 2/61 (3.27\%) patients had micrometastasis. At the time of the last follow-up, $41 \%$ of all patients were in complete disease remission and $41.1 \%$ had died from BC.

Conclusions: Our study shows that histological analysis using hematoxylin eosin (HE) method by experienced pathologists is sufficient for the diagnosis of LN metastasis and, therefore, there is no indication for routine IHC evaluation in patients at histopathological pNO stage.
\end{abstract}

Keywords: Bladder, Oncology, Tumor markers

\section{Background}

Bladder cancer $(\mathrm{BC})$ is an important public health problem with an increasing incidence rate. It is the fourth most common cancer tumor in men and the eighth in women, with an estimated 356,600 new cases diagnosed annually worldwide [1]. Radical cystectomy (RC) associated with pelvic lymphadenectomy (LND) is the standard treatment for invasive $\mathrm{BC}[2]$ and the presence of pre-operative hydronephrosis [3], perineural, and lymphatic and vascular invasion $[4,5]$ influence clinical course and prognosis.

Data from the Bladder Cancer Research Consortium (BCRC) show that $20-30 \%$ of patients at the T1 and T2

\footnotetext{
* Correspondence: iwcunha@accamargo.org.br; waltercosta@hotmail.com Pathology Department, A.C. Camargo Cancer Center, Rua Professor Antônio Prudente, 211, Sao Paulo, Sao Paulo, Brazil
}

stages at the time of $\mathrm{RC}$ will progress and many will subsequently die [6]. Lymph node (LN) metastasis is one of the main prognostic factors in $\mathrm{BC}$; however, its presence cannot be properly detected by the currently available imaging methods such as computed tomography (CT), magnetic resonance imaging (MRI), and PET-scan [7]. Additionally, the incidence of LN micrometastasis (define as metastasis less than $2 \mathrm{~mm}$ diameter) and its relevance are controversial due in part to the paucity of studies addressing the topic $[8,9]$.

As its name implies, cytokeratin AE1AE3 is a mixture of two different clones of anti-cytokeratin monoclonal antibodies, AE1 and AE3. Both of these individual clones detect certain high and low molecular weight keratins. AE1 detects the low molecular weight cytokeratins such 
as $10,14,15$, and 16 . Clone AE3 detects the high molecular weight cytokeratins $1,2,3,4,5$, and 6 , and the low molecular weight cytokeratins 7 and 8 . By combining these two reagents, a single reagent with a broad spectrum of reactivity against both high and low molecular weight cytokeratins is obtained [10]. Due to its broad reactivity, AE1AE3 has been used as a useful marker of micrometastasis in epithelial tumors [11].

The aim of our study was to use immunohistochemistry (IHC) analyses to evaluate the presence of micrometastasis and assess its prognostic role in $\mathrm{BC}$ pN0 patients submitted to RC.

\section{Methods}

In this retrospective study, a total of 63 patients were selected among the 300 sequentials BC patient records available at the Medical and Statistical Filing Service of the AC Camargo Cancer Center. The selected patients (with their paraffin blocks and slides archived in the tumor bank) had bladder urothelial tumors and were treated with $\mathrm{RC}+$ lymph node dissection (LND) at our institution between 1990 and 2009. Conventional histological evaluation indicated that these patients did not have lymph node metastasis ( $\mathrm{pN} 0$ ). The same pathologist reviewed all pN0 LN slides.

Patients who underwent neoadjuvant treatment (except local/topic BCG therapy), with non-urothelial tumor, and those whose tumor slides and/or paraffin block were not available for our study, were excluded from the analysis. There was no pNx case.

The study was approved by our institution internal Research Ethics Committee.

\section{Immunohistochemistry technique}

The sections were mounted on positively charged glass slides and dried for $30 \mathrm{~min}$ at $37{ }^{\circ} \mathrm{C}$. The sections were deparaffinized in xylene and rehydrated via a series of graded alcohols. The AE1AE3 antibody (DAKO Corporation-Denmark) was used as the immunohistochemical marker at the 1:50 dilution. All immunohistochemical procedures were performed automatically in the auto-stainer Link 48, DAKO', using the Flex Plus visualization system according to the supplier's specifications.

\section{Statistical analysis}

The statistical analysis was performed with the Windows Statistical Package for the Social Sciences (SPSS) software version 17.0. The distribution of the epidemiological, clinical, and pathological variables was presented in contingency tables; the measurement of quantitative variables was expressed by averages and standard deviations.

\section{Results}

Two of the 63 patients were excluded from the analysis after lymph node metastasis was identified in their slides after hematoxylin eosin (HE) staining pathological review, measuring $0,5 \mathrm{~mm}$ and $3,0 \mathrm{~mm}$. Another two of the 61 remaining patients had lymph nodal micrometastasis (one positive LN each patient) as revealed by the IHC analysis (Fig. 1). These 2 patients were ASA 2, had invasive tumors pT4, the average size of the lesions was $2.55 \mathrm{~cm}(2,5$ and $2.6 \mathrm{~cm}$, respectively), lymphatic and perineural invasion. Both had muscle invasive tumors in preview TUR without CIS, died from surgery complications and the average hospitalization time was 73 days (10 and 136, respectively).

The total number of removed LNs was 832, averaging 13.64 LNs per patient (total of 61 patients) (Table 1). The average follow-up time was 48 months. Hydronephrosis was present in $34.5 \%$ of the patients; $32.8 \%$ of the patients presented ASA III anesthetic risk. It was observed that nine patients $(14,8 \%)$ were operated because of the disease recurrence and $31.1 \%$ of the total number of patients showed disease progression ( $R C$ tumor stage bigger than TUR tumor stage) of Urothelial carcinoma was present in $95.1 \%$ of the cases and carcinoma in situ (CIS) in $3.3 \%$. Three patients had pT0 disease and $44.2 \%$ of the cases presented pT3-pT4 pathological stages. Vascular invasion was present in $15.4 \%$ of cases, lymphatic invasion in $36.5 \%$, and perineural invasion in $23.6 \%$. Cancer survival rates at $12,24,36,48$, and 60 months were $82.4 \%, 68 \%, 61.8 \%, 50.9 \%$, and $45.2 \%$, respectively. At the time of the last follow-up, $41 \%$ of patients were in complete $\mathrm{BC}$ remission, $3.3 \%$ had $\mathrm{BC}, 13.1 \%$ had died from other causes, $41.1 \%$ had died from BC, and $1.6 \%$ was lost to follow up (Table 2).

The two patients with LN were ASA 2 and had pT4 stage disease, showing an average tumor lesion size of $2.55 \mathrm{~cm}$ and presented lymphatic and perineural invasion. An evaluation of the impact of micrometastasis on survival was not possible because both patients who presented micrometastatic disease died from surgery complications.

\section{Discussion}

Tumor staging and grade decisively influence the treatment and prognosis of invasive BC. [12]. Therefore, tumor stage must be precisely determined in order to select the best course of treatment. Radiological LN evaluations are limited because CT scans and MRI cannot identify metastases in LNs of normal size or those minimally enlarged [7]. Pelvic lymph nodes that are larger than $8 \mathrm{~mm}$ and abdominal LNs that are larger than $10 \mathrm{~mm}$ in their longest axis are considered enlarged by CT or MRI. The sensitivity of these methods for detecting lymph node metastasis varies from 48 to $87 \%$. 


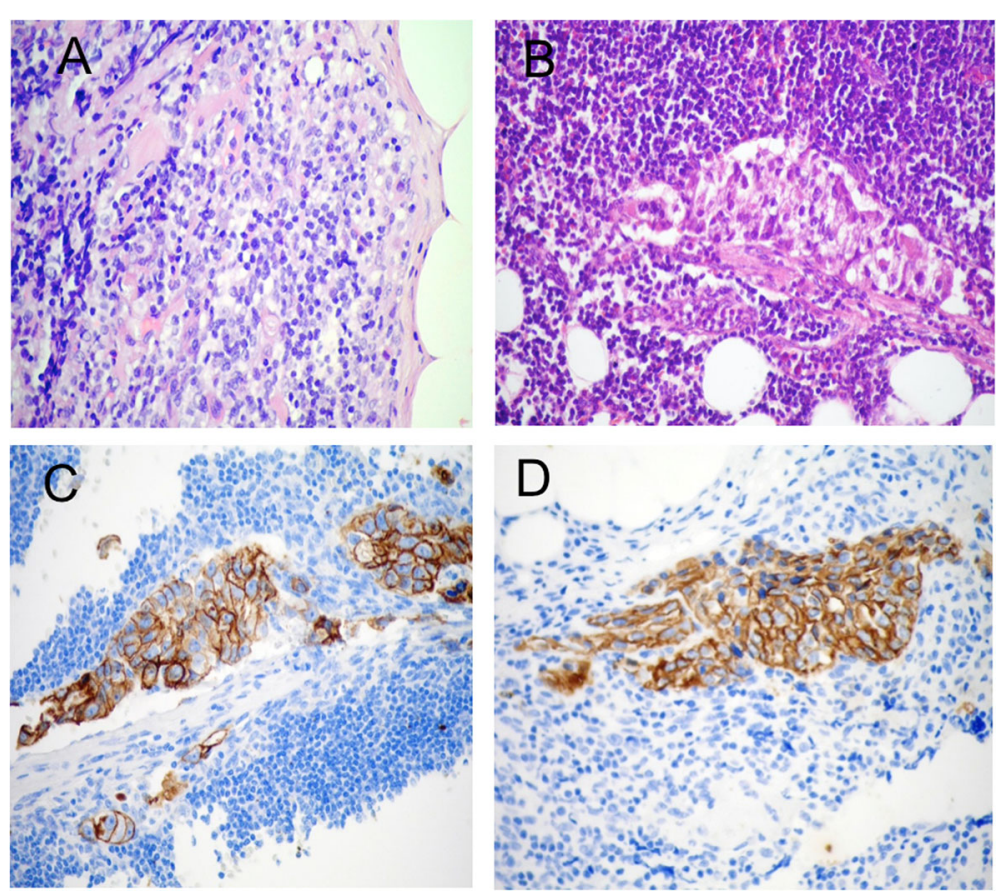

Fig. 1 Photomicrography of the lymph node tissue affected by BC metastasis. $\mathbf{a}$ and $\mathbf{b}$ : tissue stained by the hematoxylin-eosin method; $\mathbf{c}$ and $\mathbf{d}$ : tissue assessed by IHC using the AE1AE3 marker (original magnification $=400 X)$. $\mathbf{a} / \mathbf{c}$ and $\mathbf{b} / \mathbf{d}$ are from each patient described

The specificity is also low because the enlarged LN is not necessarily a marker for further neoplastic disease $[13,14]$. Currently, there is no evidence for the routine use of PET for evaluating LN staging in bladder cancer [15]. Therefore, clinical sub-staging of $\mathrm{BC}$ is a common problem. It is estimated that around $20-25 \%$ of $\mathrm{T} 1-\mathrm{T} 4$ NOM0 patients who are submitted to RC and LND present lymph nodal metastasis [16, 17], and these rates have been shown to increase with the progression of tumor staging [18]. Pelvic LND is the main LN staging method, and it has been suggested that increased lymphadenectomy extension leads to increased chances of cure [19]. LN identification can be improved by submitting the same samples in separate vials [16], by identifying specific solutions [20], and analyzing $3 \mathrm{~mm}$ sections [21].

LN micrometastasis is a marker of poor prognosis in tumors such as breast, lung, esophagus, stomach, uterus, and melanoma. SERAJ et al. studied $27 \mathrm{BC}$ patients within Ta-T3N0 clinical stages. The biopsies of the largest

Table 1 LN status in samples from 61 BC patients at pNO stage

\begin{tabular}{llll}
\hline Variable & Category & N & $(\%)$ \\
\hline Lymph node metastasis & Absent & 59 & 96.73 \\
& AE1AE3 + Marker & 2 & 3.27 \\
Total number of LND & & 832 & $100 \%$ \\
LND average number & & $13.64(2-52)$ & \\
LND number & Up to 14 & 40 & 65.60 \\
& $>14$ & 21 & 34.40 \\
\hline
\end{tabular}

tangible removed LNs were evaluated through RT-PCR for the presence of uroplakin II (UPII) mRNA. A total of $17 \%$ of the LNs from pT2N0 patients and 67\% from pT3N0 patients presented lymph nodal micrometastasis [8]. RETZ et al. found $29 \%$ positivity when investigating the use of mucin 7 (MUC-7) expression in the detection of micrometastasis in histologically normal LNs [9]. The expression of cytokeratin 19 (CK19) and uroplakin II (UPII) were used in the evaluation of micrometastasis in 760 LNs from 40 patients who underwent RC with LND. Histopathological review detected 29 positive LNs in six patients; however, CK19 and UPII positive expression indicated the presence of lymph nodal metastasis in 49 LNs and 98 LNs, respectively. Patients with micrometastasis detected by these biomarkers showed poorer survival rates when compared with patients without micrometastasis, regardless of the presence of histologically positive LNs [22]. Recently, MARIN-AGUILERIA et al. analyzed the role of the expression of 5 genes in identifying LN micrometastasis using RT-PCR. The combined expression of two genes (FXYD3 and KRT20) differentiated the positive LNs from the controls (healthy) with $100 \%$ sensitivity and specificity. These authors showed that the expression of two genes identified positive LNs in $20.5 \%$ of the pN0 patients evaluated histologically [23].

YANG et al. [11] were the first to evaluate the immunohistochemical role of the AE1AE3 antibody in detecting micrometastasis in LNs in 229 LNs (pN0) from 19 invasive $\mathrm{BC}$ patients submitted to $\mathrm{RC}$. Three sections were 
Table 2 Patients and pathological characteristics

\begin{tabular}{|c|c|}
\hline Variable & N (\%) \\
\hline Follow-up (months) & $48(1-249)$ \\
\hline Age (mean) & $66(39-8)$ \\
\hline \multicolumn{2}{|l|}{ Smoking } \\
\hline No & $20(32.7)$ \\
\hline Yes & $41(67.2)$ \\
\hline \multicolumn{2}{|l|}{ Hydronephrosis } \\
\hline No & $36(59.0)$ \\
\hline Yes & $25(41.0)$ \\
\hline \multicolumn{2}{|l|}{ ASA classification } \\
\hline 1 & $4(6.5)$ \\
\hline 2 & $36(59.0)$ \\
\hline 3 & $21(34.5)$ \\
\hline \multicolumn{2}{|l|}{ Histology } \\
\hline Urothelial carcinoma & $58(95,1)$ \\
\hline $\mathrm{CIS}$ & $2(3.1)$ \\
\hline Without Tumor & $1(1,6)$ \\
\hline \multicolumn{2}{|l|}{ pT stage } \\
\hline TO & $3(4,9)$ \\
\hline Tis & $2(3.2)$ \\
\hline $\mathrm{Ta}$ & $6(9.8)$ \\
\hline $\mathrm{T} 1$ & $9(14.8)$ \\
\hline $\mathrm{T} 2$ & $16(26.2)$ \\
\hline Т3а & $8(13.1)$ \\
\hline $\mathrm{T} 3 \mathrm{~b}$ & $7(11.5)$ \\
\hline $\mathrm{T} 4$ & $12(19.7)$ \\
\hline \multicolumn{2}{|l|}{ Histological grade } \\
\hline Low & $18(31.0)$ \\
\hline High & $40(69.0)$ \\
\hline \multicolumn{2}{|l|}{ Pathologic grade } \\
\hline 0 & $3(4,9)$ \\
\hline 1 & $6(9,8)$ \\
\hline 2 & $12(19,7)$ \\
\hline 3 & $26(42,6)$ \\
\hline 4 & $14(26)$ \\
\hline \multicolumn{2}{|l|}{ Vascular invasion ${ }^{\mathrm{a}}$} \\
\hline No & 44 (84.6) \\
\hline Yes & $8(15.4)$ \\
\hline \multicolumn{2}{|l|}{ Lymphatic invasion $^{a}$} \\
\hline No & $33(63.4)$ \\
\hline Yes & $19(36.5)$ \\
\hline \multicolumn{2}{|l|}{ Perineural invasion ${ }^{a}$} \\
\hline No & $26(76.4)$ \\
\hline Yes & $8(23.6)$ \\
\hline
\end{tabular}

Table 2 Patients and pathological characteristics (Continued)

\begin{tabular}{ll}
\hline Disease progression & \\
No & $42(68.9)$ \\
Yes & $19(31.1)$ \\
Cancer Survival & \\
$12 \mathrm{~m}$ & $(82,4)$ \\
$24 \mathrm{~m}$ & $(68)$ \\
$36 \mathrm{~m}$ & $(61,8)$ \\
$48 \mathrm{~m}$ & $(50,9)$ \\
$60 \mathrm{~m}$ & $(45,2)$
\end{tabular}

${ }^{\text {aExcluding missing cases }}$

prepared for IHC, and one was stained by HE from each tissue block. The antibodies used in the IHC evaluation were CAM 5.2 and AE1AE3. Only one LN was positive for CAM 5.2. However, this micrometastasis could be observed through a more thorough analysis in a deeper section stained by HE. The authors concluded that LN analysis with $\mathrm{HE}$ method is adequate and has a better cost-benefit ratio compared to IHC for identifying metastasis in the pelvic LNs of $\mathrm{BC}$ patients. Conversely, $\mathrm{ABE}$ et al. [24] recently analyzed the role of AE1AE3 in the diagnosis of micrometastasis in LNs from patients with upper urinary tract urothelial carcinoma who underwent nephroureterectomy associated with retroperitoneal LND. Seven hundred and fourteen LNs from 51 patients were reviewed with the HE method and analyzed for AE1AE3 reaction. Micrometastasis was identified in 7 patients (13.7\%). No difference in survival was observed between patients with and without micrometastasis after 45 months of follow-up. In our study, only 2 patients considered as pNO in the conventional histological evaluation showed lymph nodal micrometastasis. Survival analysis was not possible since both patients died from surgical complications during hospitalization. We observed that since 1996, the number of surgically dissected LNs has increased substantially, reaching an average of 20 LNs per patient in the last decade. Despite the dissection of many LNs and consequently, the increased number of LN analyses, none of them were positive for AE1AE3 in the IHC evaluation.

The finding of epithelial cells in the LNs, whether by IHC or the biomolecular method, does not necessarily indicate the occurrence of metastases, and thus, the morphological evaluation remains necessary. Benign epithelial inclusions represent ectopic foci of embryonic epithelium in the Müllerian ducts (paramesonephric ducts) that can be found in the lymph nodes, peritoneum pelvic, greater omentum, uterine ligaments, tubes, and ovaries. The exact frequency with which these inclusions are found in the pelvic and peri-aortic LNs is uncertain, ranging from 1 to $40 \%$ in the literature [25]. The importance of knowing the benign epithelial inclusions 
lies in the fact that they can be easily mistaken as metastatic foci.

Our data corroborate the results reported by YANG et al. [11], who concluded that a thorough histological analysis using the $\mathrm{HE}$ method by experienced $\mathrm{BC}$ pathologists is sufficient for the diagnosis of lymph nodal metastasis and, therefore, no indication for routine IHC evaluation in patients at pN0 stage is indicated. In addition, we believe that the $\mathrm{LN}$ analysis through a single section is sufficient for the diagnosis of lymph nodal metastasis. In conclusion, we do not recommend the indication of routine IHC evaluations of LNs of surgically treated pNO BC patients.

\section{Conclusions}

Our study shows that histological analysis using hematoxylin eosin (HE) method by experienced pathologists is sufficient for the diagnosis of LN metastasis and, therefore, there is no indication for routine IHC evaluation in patients at histopathological pN0 stage.

\section{Abbreviations}

BC: Bladder cancer; BCRC: Bladder Cancer Research Consortium;

CIS: Carcinoma in situ; CT: Computed tomography; HE: Hematoxylin eosin; IHC: Immunohistochemistry; LN: Lymph node; LND: Lymph node dissection; LNs: Lymph nodes; MRI: Magnetic resonance imaging; RC: Radical cystectomy; SPSS: Statistical package for the social sciences

\section{Acknowledgements}

None declared.

Funding

None declared.

\section{Availability of data and materials}

The datasets during and/or analysed during the current study available from the corresponding author on reasonable request.

\section{Authors' contributions}

GC: design, concept, acquisition of data, analysis and interpretation of data. WC: revising and drafting of the manuscript. CP: analysis and interpretation of data. CS: revising. FF: design, concept and analysis of data. IC: interpretation of data, revising and drafting the manuscript. GG: interpretation of data and revising. All authors read and approved the final manuscript.

\section{Competing interests}

The authors declare that they have no competing interests.

\section{Consent for publication}

Not applicable.

\section{Ethics approval and consent to participate}

The study was approved by our institution internal Research Ethics Committee. Comitê de Ética em Pesquisa (cep-hcancer@accamargo.org.br).

Received: 8 July 2016 Accepted: 15 November 2016

Published online: 15 December 2016

\section{References}

1. Abdel-Latif M, Abol-Enein H, El-Baz M, Ghoneim MA. Nodal involvement in bladder cancer cases treated with radical cystectomy: incidence and prognosis. J Urol. 2004;172:85-9.

2. Stenzl A, Cowan NC, De Santis M, et al. Update of the Clinical Guidelines of the European Association of Urology on muscle-invasive and metastatic bladder carcinoma. Actas Urol Esp. 2010;34:51-62.
3. Kin HY, Wang SZ, Chen JX, Chen LW, Xiao J. The prognostic value of hydronephrosis in bladder cancer treated by radical cystectomy. Urologia. 2011;78:17-21.

4. Bolenz C, Herrmann E, Bastian PJ, et al. Lymphovascular invasion is an independent predictor of oncological outcomes in patients with lymph node-negative urothelial bladder cancer treated by radical cystectomy: a multicentre validation trial. BJU Int. 2010;106:493-9.

5. Hugen CM, Polcari AJ, Fitzgerald MP, Dauw C, Flanigan RC, Quek ML. Risk factors for recurrence following radical cystectomy for pathologic node negative bladder cancer. J Surg Oncol. 2010;102:334-7.

6. Shariat SF, Karakiewicz PI, Palapattu GS, et al. Outcomes of radical cystectomy for transitional cell carcinoma of the bladder: a contemporary series from the Bladder Cancer Research Consortium. J Urol. 2006;176:2414-22. discussion 22.

7. Yang WT, Lam WW, Yu MY, Cheung TH, Metreweli C. Comparison of dynamic helical CT and dynamic MR imaging in the evaluation of pelvic lymph nodes in cervical carcinoma. AJR Am J Roentgenol. 2000;175:759-66.

8. Seraj MJ, Thomas AR, Chin JL, Theodorescu D. Molecular determination of perivesical and lymph node metastasis after radical cystectomy for urothelial carcinoma of the bladder. Clin Cancer Res. 2001:7:1516-22.

9. Retz M, Lehmann J, Szysnik C, et al. Detection of occult tumor cells in lymph nodes from bladder cancer patients by MUC7 nested RT-PCR. Eur Urol. 2004:45:314-9.

10. Miettinen M. Immunohistochemistry of soft tissue tumours - review with emphasis on 10 markers. Histopathology. 2014;64(1):101-18.

11. Yang XJ, Lecksell K, Epstein II. Can immunohistochemistry enhance the detection of micrometastases in pelvic lymph nodes from patients with highgrade urothelial carcinoma of the bladder? Am J Clin Pathol. 1999;112:649-53.

12. Jewett HJ. Proceedings: Cancer of the bladder: diagnosis and staging. Cancer. 1973;32:1072-4.

13. Dorfmann RE, Alpern MB, Gross BH, Sandler MA. Upper abdominal lymph nodes: criteria for normal size determined with CT. Radiology. 1991;180:319-22.

14. Barentsz JO, Engelbrecht MR, Witjes JA, LA Rosette JJ, Van Der Graaf M. MR imaging of the male pelvis. Eur Radiol. 1999:9:1722-36.

15. Swinnen G, Maes A, Pottel H, et al. FDG-PET/CT for the preoperative lymph node staging of invasive bladder cancer. Eur Urol. 2010:57:641-7.

16. Stein JP, Penson DF, Cai J, et al. Radical cystectomy with extended lymphadenectomy: evaluating separate package versus en bloc submission for node positive bladder cancer. J Urol. 2007;177:876-81. discussion 81-2.

17. Bellmunt J, Albiol S, Suarez C, Albanell J. Optimizing therapeutic strategies in advanced bladder cancer: update on chemotherapy and the role of target agents. Crit Rev Oncol/Hematol. 2009;69:211-22.

18. Lotan Y. Role of biomarkers to predict outcomes and responses to therapy. Urol Oncol. 2010;28:97-101.

19. Karl A, Carroll PR, Gschwend JE, et al. The impact of lymphadenectomy and lymph node metastasis on the outcomes of radical cystectomy for bladder cancer. Eur Urol. 2009:55:826-35.

20. Koren R, Paz A, Lask D, et al. Lymph-node revealing solution: a new method for detecting minute lymph nodes in cystectomy specimens. Br J Urol. 1997;80:40-3

21. Herr HW. Pathologic evaluation of radical cystectomy specimens. Cancer. 2002;95:668-9.

22. Kurahashi T, Hara I, Oka N, Kamidono S, Eto H, Miyake H. Detection of micrometastases in pelvic lymph nodes in patients undergoing radical cystectomy for locally invasive bladder cancer by real-time reverse transcriptase-PCR for cytokeratin 19 and uroplakin II. Clin Cancer Res. 2005; 11:3773-7.

23. Marin-Aguilera M, Mengual $L$, Burset $M$, et al. Molecular lymph node staging in bladder urothelial carcinoma: impact on survival. Eur Urol. 2008;54:1363-72.

24. Abe T, Shinohara N, Muranaka M, et al. Role of lymph node dissection in the treatment of urothelial carcinoma of the upper urinary tract: multiinstitutional relapse analysis and immunohistochemical re-evaluation of negative lymph nodes. Eur J Surg Oncol. 2010;36:1085-91.

25. Reich O, Tamussino K, Haas J, Winter R. Benign mullerian inclusions in pelvic and paraaortic lymph nodes. Gynecol Oncol. 2000;78:242-4. 